\section{NO: more muscles}

\author{
By Kai-Jye Lou, Staff Writer
}

Researchers at the University of Manitoba and the Carolinas Medical Center have uncovered myogenesis-promoting properties in an over-thecounter expectorant and have boosted the effect by synthesizing an analog that also releases the myogenic booster nitric oxide. ${ }^{1}$ The next step will be to see if these effects translate into a meaningful therapeutic benefit in animal models of muscular dystrophy and atrophy.

The Manitoba team, led by Judy Anderson, had previously shown that nitric oxide (NO) activates quiescent satellite precursor cells in skeletal muscle, which generate new precursor cells for muscle growth and repair. ${ }^{2}$ Anderson, who heads the Department of Biological Sciences at the university, has also shown that increased NO levels in both normal and dystrophic muscle can promote regeneration., ${ }^{2,3}$

In a paper in Molecular Pharmaceutics, Anderson and colleagues now report the synthesis and preliminary evaluation of guaifenesin dinitrate, a compound that delivers NO to skeletal muscle and can be given orally or transdermally.

The parent compound guaifenesin is available over the counter to help clear phlegm from the respiratory tract. The compound also has muscle relaxant properties.

In adult mice, a transdermal ointment formulation of guaifenesin dinitrate increased DNA synthesis - an indicator of muscle satellite cell activation and proliferation-in the back and quadriceps muscles compared with what was seen using placebo. Mice given an oral formulation of the compound showed greater satellite cell activation and myoblast production than oil-treated controls.

"The fact that they showed a 30-50\% increase in DNA synthesis in the muscle satellite cells was very interesting and this clearly suggests efficacy," said Ryszard Kole, SVP of discovery research at AVI BioPharma Inc., which has compounds in Phase Ib and preclinical development for Duchenne muscular dystrophy (DMD).

The academics also showed that the muscle relaxant methocarbamol-an analog of guaifenesin-can promote muscle proliferation on its own, and that this effect was additive with NO.

Anderson told SciBX that the formulation is the first of a new family of NO donor molecules that boosts the myogenesis-promoting effects of muscle relaxants via NO.

"Previous work has demonstrated the capability of nitric oxide, possibly via a pharmacological action on the regulatory protein follistatin, to induce satellite cell myogenesis," noted Csaba Szabo,
CSO and SVP at Ikaria Holdings Inc. "The current work builds upon these data and demonstrates that both the classical NO donor compound, isosorbide dinitrate, and a novel NO donor compound synthesized by the authors, guaifenesin dinitrate, are able to produce satellite cell myogenesis in the skeletal muscle of mice."

Ikaria markets INOmax inhaled nitric oxide to treat persistent pulmonary hypertension in newborns.

Looking forward, Ellen Welch, associate director of biology at PTC Therapeutics Inc., suggested that "this may be a good starting point to identify and develop muscle-specific nitric oxide-based therapies."

PTC's ataluren, which is partnered with Genzyme Corp., is a small molecule that facilitates the complete translation of proteins containing nonsense mutations. The compound is in a pivotal Phase IIb trial to treat the $15 \%$ of DMD patients with a nonsense mutation in the gene encoding dystrophin. Ataluren has also completed a Phase IIa trial in cystic fibrosis (CF).

\section{A combinatorial approach}

Anderson thinks the myogenesis-promoting effects of NO-donating compounds like guaifenesin dinitrate would be most applicable in muscular dystrophy and age-related muscular atrophy.

$\mathrm{DMD}$ is caused by mutations in the dystrophin gene that result in the loss of protein function. The loss of dystrophin results in muscle damage and eventual muscle necrosis and leads to premature death. ${ }^{4}$ For the former indication, Anderson thinks the compounds probably would have an adjuvant role.

Kole agreed. "In muscle-wasting diseases other than DMD, the myogenesis-promoting properties of the NO-donating compound may be sufficient, but I don't think this would be viable in the long run as a stand-alone approach for DMD. The reason is because even if you increase myogenesis in patients with DMD, these new muscle cells will still produce nonfunctional dystrophin."

"While a molecule that modulates muscle growth and regeneration will not cure DMD, the fact that it will delay the progression of the disease is still very significant," added Welch.

With regard to atrophy, Anderson thinks that a transdermal NObased treatment targeted to skeletal muscle would be useful as a complement to exercise.

Indeed, the benefits and risks associated with each mode of delivery will need to be carefully assessed in future studies.

Guaifenesin dinitrate's systemic availability is important, noted Kole, because in certain musculoskeletal diseases like DMD, all muscles in the body are affected. "This means a candidate therapeutic approach will need to be able to demonstrate efficacy via systemic delivery," he said.

Conversely, because NO exerts a broad range of pharmacological effects on various biological systems, targeting its delivery to a specific region or tissue could help decrease side effects. 


\section{TARGETS \& MECHANISMS}

\section{Translating forward}

An unanswered question is whether guaifenesin dinitrate's myogenesis-promoting effects in mice will translate into a meaningful improvement in disease symptoms.

"The main caveat I have is that the researchers only showed the myogenic effects in normal mice," Kole told SciBX. "I will feel much better about these findings when the group also shows these results in the context of a disease model like the $m d x$ mouse, either as a stand-alone treatment or in combination with another approach."

Szabo said it will be important to show the compound's specificity for skeletal muscle. "The authors did not show regional distribution of the guaifenesin dinitrate compound. It is unclear whether the parent compound and/or the NO released from it will enter tissues other than skeletal muscle" and produce known side effects of NO such as systemic vascular relaxation and a fall in blood pressure, he said.

In addition, Szabo said the study only looked at the number of satellite cells induced by guaifenesin dinitrate. "The studies did not investigate whether this actually translates into detectable increases in muscle mass, muscle strength and other relevant functional parameters," he said.

Welch said she would like to see guaifenesin dinitrate evaluated in longer term safety and efficacy studies, given that it is likely to be used chronically. She also wanted to see how long the effects of the NO-donating compound will last in muscle, which would provide insights into a dosing schedule.

Anderson said her group has preliminary data suggesting that guaifenesin dinitrate promotes muscle regeneration in the $m d x$ mouse model of muscular dystrophy. She added that her group is continuing with studies to evaluate the compound in both muscular atrophy and additional muscular dystrophy models.

According to Anderson, a patent application has been filed covering compositions and methods for increasing NO delivery to promote muscle growth and repair under normal and diseased states. She said the university has transferred the rights to the inventors, which include herself, Gu Qi Wang and Frank Burczynski. The work is available for licensing.

Wang is a research faculty member in the McColl-Lockwood Laboratory for Muscular Dystrophy Research at the Carolinas Medical Center. Burczynski is a professor in the faculty of pharmacy at the University of Manitoba.

Lou, K.-J. SciBX 2(15); doi:10.1038/scibx.2009.607

Published online April 16, 2009

\section{REFERENCES}

1. Wang, G. et al. Mol. Pharm.; published online March 24, 2009; doi: $10.1021 / \mathrm{mp} 800226 z$

Contact: Judy E. Anderson, University of Manitoba, Winnipeg, Manitoba, Canada

e-mail: janders@ms.umanitoba.ca

2. Anderson, J.E. Mol. Biol. Cell 11, 1859-1874 (2000)

3. Anderson, J.E. \& Vargas, C. Neuromuscul. Disord. 13, 388-396 (2003)

4. Hoffman, E. et al. Cell 51, 919-928 (1987)

COMPANIES AND INSTITUTIONS MENTIONED

AVI BioPharma Inc. (NASDAQ:AVII), Portland, Ore.

Carolinas Medical Center, Charlotte, N.C.

Genzyme Corp. (NASDAQ:GENZ), Cambridge, Mass.

Ikaria Holdings Inc., Clinton, N.J.

PTC Therapeutics Inc., South Plainfield, N.J.

University of Manitoba, Winnipeg, Manitoba, Canada 